\title{
EL PUNTO DE ENCUENTRO DE LA TERAPIA FAMILIAR Y LA EDUCACIÓN FAMILIAR
}

Gabriela Esponda Jones

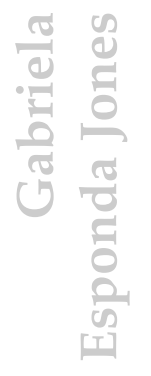

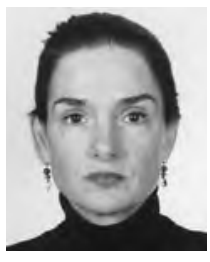

Maestría en Psicología Clínica, Newport University.

Licenciatura en Psicología, Universidad Intercontinental.

Actualmente proporciona consulta psicoterapéutica para adultos, pareja y familia, e imparte Talleres para Padres en el ÍTEMS.

Correo electrónico:

[espondaquintanar@yahoo.com.mx].

\section{RESUMEN}

El presente artículo muestra cómo las aportaciones de la cibernética y la terapia estructural pueden brindar a la educación familiar elementos útiles en su labor preventiva. Con base en la cibernética, este trabajo analiza la manera en la que la familia se retroinforma (positiva o negativamente) a sí misma para mantenerse como tal. En cuanto a la terapia estructural, se mencionan los subgrupos que deben conformarla (subsistema conyugal, parental y fraternal), como índices de normalidad y funcionamiento sano.

Palabras clave: cibernética; sistema; subsistema; conflicto; familia; terapia estructural; educación familiar; reglas; retroinformación; cambio. 


\section{ABSTRACT}

Family Education in its preventive task can benefit from two important pillars of Family Therapy. Cybernetics is the first pillar, and it's concept of the importance of (negative or positive) feedback to keep the family system working as such. The second pillar is the conceptualization given by Structural Therapy as a system composed by subsystems (marital, paternal and filial). These two sets of ideas can help the Family Educator to form a concept of family normality in order to orient his work.

Key words: Cybernetics; system; subsystem; conflict; family; structural therapy; family education; rules; feedback; change.

\section{INTRODUCCIÓN}

La psicología y la pedagogía tienen en común el interés por el perfeccionamiento de la persona. Ambas ciencias poseen aportaciones importantísimas que deben hacerse mutuamente.

En el presente escrito, se analizan dos diferentes pilares de la psicoterapia breve y sus posibles conexiones con el terreno de la educación familiar.

\section{CIBERNÉTICA}

Durante la Segunda Guerra Mundial, la Fundación Josiah Macy, patrocinó una serie de conferencias de carácter interdisciplinario, en las cuales participaron matemáticos, ingenieros y científicos sociales prominentes.

Las conferencias versaron acerca del estudio de la comunicación en referencia al control y la regulación (por ejemplo, de misiles y cohetes a través de mecanismos de retroinformación). Uno de estos científicos, Norbert Weiner, acuñó el término cibernética ${ }^{4}$ para describir dichos procesos. La cibernética es el estudio de las formas mediante las cuales un 
sistema se autocorrige a sí mismo para mantenerse en equilibrio o conducirse hacia una meta determinada. Esto lo hace especialmente a través del flujo de información que él mismo emite y recibe.

En términos cibernéticos, se considera que la retroinformación puede ser positiva o negativa, según el efecto de desviación o acercamiento de la meta que éste dé al sistema. La retroinformación negativa es la que reduce o evita la desviación o el cambio, mientras que la retroinformación positiva aleja al sistema de su estado deseado ${ }^{5}$.

Algunos investigadores de las ciencias sociales como Gregory Bateson, en 1972, encontraron que existe relación entre la teoría cibernética y la psicología, resumiéndola de la siguiente manera: «Todos los cambios pueden ser entendidos como un esfuerzo para mantener la constancia y toda constancia se mantiene a través del cambio» ${ }^{6}$.

El concepto de cibernética, inició una nueva era en la psicoterapia. En primera instancia, el motivo de la disfuncionalidad de la conducta humana, dejó de ser vista como un conflicto intrapsíquico para empezar a entenderse como producto de un sistema, esto es, la familia.

A la luz de la cibernética, el paralelismo entre sistemas máquinas y sistemas familias, podría resumirse así ${ }^{7}$ :

1. Las reglas familiares moderan el rango de tolerancia que el sistema familiar está dispuesto a aceptar.

2. El mecanismo de retroinformación negativa que generalmente utiliza la familia, es la puesta en práctica de sus reglas.

3. Cuando los mecanismos de retroinformación negativa son inefectivos, la familia recurrirá a mecanismos de retroinformación positiva en busca de alternativas que funcionen. 
Estas ideas aportaron cambios significativos al campo de la psicoterapia. Entre ellos, como se ha mencionado antes, el individuo empezó a ser visto como parte integrante y resultante de un sistema. El conflicto comenzó a interpretarse como un mal funcionamiento del sistema de comunicaciones de la familia y no como un choque de personalidades. Finalmente, este cambio de enfoque, brindó la posibilidad de circunscribir los objetivos terapéuticos a metas concretas que podían reducir el tiempo del tratamiento terapéutico, dando lugar a los primeros esbozos de la psicoterapia breve.

\section{TERAPIA ESTRUCTURAL}

Entre las escuelas más influyentes de la psicoterapia breve se encuentra la corriente estructural de Salvador Minuchin ${ }^{8}$, cuya postura fue planteada en 1974 mediante la publicación de su libro Familias y terapia familiar. Este autor considera que la familia normal no puede ser diferenciada de la disfuncional por la ausencia de conflictos: todas tienen problemas. La diferencia radica en la manera como la familia se estructura para enfrentarlos.

El modelo de familia que Minuchin propone se caracteriza por subsistemas que operan con límites claros:

1. Subsistema conyugal: Formado por los esposos, quienes pierden cierto grado de individualidad para construir un sentido de pertenencia mutua que los ayude a operar colaborativamente. Es importante que este subsistema esté protegido por límites claros y que evite la invasión de otros sistemas.

2. Subsistema parental: Constituido por el padre y la madre. Debe permitir el acceso de los hijos a ambos padres, sin incluirlos en el subsistema conyugal. Su funcionamiento eficaz requiere que los padres y los hijos acepten el hecho de que existe un uso diferenciado de la autoridad. 
3. Subsistema fraterno: Es el laboratorio social en el cual los hermanos aprenden a relacionarse con sus iguales. Este aprendizaje será aplicado más tarde en el mundo externo.

El objetivo de la terapia estructural es que la familia que acude a terapia regrese a una estructura jerarquizada (descrita en los puntos anteriores), cuando dicho orden se haya alterado.

\section{PUNTO DE ENCUENTRO DE EDUCACIÓN FAMILIAR Y TERAPIA FAMILIAR}

\section{Cibernética y Educación Familiar}

La labor preventiva de la educación familiar puede servirse de la labor curativa de la terapia familiar. Una de las aportaciones fundamentales de la teoría que sustenta a la terapia familiar, es que lo que la familia hace, dice y es, es decisivo para la calidad de vida de sus integrantes.

Ante el desajuste social y familiar que presenciamos cotidianamente, surgen dudas acerca de lo que está sucediendo al interior de las familias jóvenes. Pensando en ellas en términos cibernéticos y, por lo tanto, ubicándolas como sistemas que se autocorrigen a través de sus propias reglas, surgen los siguientes cuestionamientos:

¿Qué características poseen las reglas familiares en la actualidad? ¿Los sistemas familiares de hace algunas décadas, establecían reglas claras? ¿Esto las llevaba a un funcionamiento más adecuado? ¿Predominaba anteriormente la retroinformación negativa? ¿Predomina actualmente la retroinformación positiva? ¿Abarcan dichas reglas todas las necesidades que, como personas, tienen los integrantes del sistema? ¿Se está retroalimentando el sistema? ¿Está totalmente desgobernado por falta de información?

Para tratar de proporcionar respuesta a estas interrogantes, es fundamental entender que la familia actual está inmersa en las consecuencias históricas del feminismo. La mujer ha dejado las labores del hogar y la crianza de los hijos como única actividad y ha salido al mundo 
laboral, económico y político. Esto ha dado lugar a que los matrimonios compartan responsabilidades en todos los niveles: económico, educativo, afectivo ${ }^{9}$.

En mi experiencia, la familia se encuentra aún en una etapa de transición en la que todavía no ha podido definir un modo actual y propio de ser. Las modificaciones en la relación hombre-mujer han hecho que, frecuentemente, las parejas centren más su atención en la definición de ellos como individuos y como pareja. Al concentrar su interés y energía en definirse como individuos e integrantes de una pareja, le han restado atención a su rol de padres, dejando a sus hijos un tanto desprotegidos.

Esto permite que el sistema familiar no se esté retroinformando de manera completa. Existen partes esenciales del sistema familiar que están quedando en el vacío. Las consecuencias de éste, las contemplamos todos los días en una infancia y juventud enfrentadas a la vida con una conciencia endeble, un control pobre de sus impulsos, una serie de creencias desviadas y una sensibilidad moral subdesarrollada ${ }^{10}$.

El sistema familiar, encabezado por los padres, debe encontrar un nuevo mapa que proporcione a todos — pero especialmente a los hijos-, herramientas para convertirse en constructores de una vida personal y social sólida. Estas herramientas deben adquirirse para beneficio propio y de las personas que formen parte de sus vidas.

En mi experiencia, la parte del sistema familiar que se está quedando carente de cualquier tipo de retroinformación, es la conciencia moral. Michelle Borba, autora del libro Building Moral Intelligence, considera que la inteligencia moral se construye a través de estas siete virtudes:

- La empatía: Actitud que permite salirnos de nuestra propia realidad para "ponernos en los zapatos del otro», sensibilizándonos frente a sus necesidades y dificultades. La empatía es la precursora de la generosidad, virtud que hoy, tristemente, ha sido desplazada por el individualismo.

M. Villalobos, «Los fundamentos esenciales de la educación familiar», Revista Panamericana de Pedagogía, p. 255-271.

${ }^{10}$ M. Borba, Building Moral Intelligence, p. 316. 
- La conciencia: Voz interna que aprende a tomar decisiones informadas al enfrentarse a las tentaciones. Su formación es vital pues es una brújula que en todo momento ayuda a elegir el camino del bien sobre el del mal.

- El autocontrol: Implica el aprendizaje del control de los impulsos; al hacerlo, la persona crea un espacio de reflexión en el cual, además de su beneficio personal, puede incluir el bienestar de otros. Asimismo, la persona que se autocontrola goza de un beneficio adicional: adquiere la certeza de que puede confiar en sí misma y que su andar está en buenas manos.

- El respeto: Es la actitud que resulta de reconocer al otro como valioso y digno. En la actualidad, muchos niños no han sido informados acerca de la valía que poseen todas las personas, a pesar de no ser iguales. En múltiples ocasiones, la poca y mala información que el sistema se proporciona a sí mismo, lleva a que los niños desconozcan la noción de respeto.

- La enseñanza de la bondad: Permite a la persona sentir compasión por los demás y también impulsa a ser pro activo. La bondad es la compasión llevada al acto a través del cual se le tiende la mano al otro.

- El aprendizaje de la justicia: Ayuda a la persona a medirse a sí misma de una manera objetiva y crítica, al mismo tiempo en el que se aprende a ser imparcial y equitativo en todas las situaciones de la vida.

- La práctica de la tolerancia: Abre la posibilidad de apreciar las cualidades del otro y extiende el pensamiento a diversas perspectivas y creencias.

\section{Terapia Estructural y Educación Familiar}

Los cambios sociales de las últimas décadas han transformado también a la familia. Tan sólo en la generación de nuestros abuelos, los padres solían ser figuras enormes, dueñas de toda autoridad, que controlaban a sus hijos con su mera presencia.

Las generaciones posteriores a la liberación femenina, buscaron «evolucionar» hacia la igualdad. Ante el temor de ser gigantescos y represores, 
los padres se convirtieron en una especie de «amigos» que, para eludir sentimientos de culpa, propician que sus hijos se desarrollen en un clima de «libertad». Sin embargo, la realidad es que muchas personas educadas bajo este criterio, han crecido en el vacío.

Jaime Barylko, filósofo y educador argentino, afirma que:

En ese clima nos criamos, en el de los padres culpables y el de los hijos absueltos a priori. Y es cierto, los padres son culpables, culpables de hacerse culpables. Culpables del miedo, el miedo a los hijos. Culpables de usar el miedo para eludir responsabilidades de educación, decisión, formación en valores. Culpables de no ser padres o serlo únicamente a la defensiva, pidiendo perdón, solicitando un mendrugo de caridad comunicativa, comprendiendo demasiado, cuando no comprenden nada ${ }^{11}$.

La falta de una autoridad familiar clara, es decir, de un subsistema parental sólido, ha hecho que la jerarquía familiar se invierta. En muchas ocasiones, los hijos, aun siendo pequeños, ocupan la silla del poder y sus padres se doblegan ante sus demandas. Los límites se han contaminado y los hijos -inconscientemente atemorizados por el poder que detentan- se vuelven agresivos: están especialmente enojados con sus padres.

Las ideas que sustenta la terapia estructural poseen una aportación fundamental para la educación familiar. La familia sana, funciona cuando los subsistemas existen como tales, con límites claros que evitan la contaminación de unos con otros. La familia produce hijos sanos cuando se presta para ser un laboratorio de aprendizaje. El lugar para aprender a entender y manejar las relaciones disparejas de poder, es la familia.

El subsistema parental tiende a fundirse con el subsistema fraterno. Para muchos, sin darse quizá cuenta de ello, el hecho de abandonar el ejercicio de la autoridad, implica desentenderse del difícil trabajo de supervisión del cumplimiento de las directivas familiares. De esta manera se crea, entre otras cosas, la ilusión de una juventud perdurable y de una familia sana que opera como una comunidad de iguales. 


\section{CONCLUSIÓN}

La educación familiar en su labor preventiva, puede hacer uso de algunas contribuciones de la terapia familiar, entre otras, se hallan la cibernética y la terapia estructural que aportan parámetros para establecer la salud y funcionalidad en la familia.

La cibernética ayuda a comprender la manera en la que la familia se retroalimenta para conservarse como tal; la terapia estructural brinda un esquema para seguir y ubicar - mapear - a la familia en subsistemas que, si operan con fronteras claras pero permeables, mantendrán a la familia funcionando de manera sana.

\section{BIBLIOGRAFÍA}

- BARYLKO, J., El miedo a los hijos, Emecé, Argentina, 1997, 214 p.

- BATESON, G., Steps to an Ecology of the Mind, Dutton, New York, 1972, $381 \mathrm{p}$.

- BORBA, M., Building Moral Inteligence, Jossey-Bass, USA, 2001, 316 p.

- GOldenberG, I. \& GOLDENBERG, H., Family Therapy an Overview, Brooks / Cole, USA, 2000, 492 p.

- NICHOLS, M. \& SHWARTZ, R., Family Therapy Concepts and Methods, Allyn and Bacon, USA, 2001, 570 p.

- MINUCHIN, S., Familias y terapia familiar, Gedisa, Barcelona, 1995, $349 \mathrm{p}$.

- VILLALOBOS, M., «Los fundamentos esenciales de la educación familiar», Revista Panamericana de Pedagogía, núm. 3, Facultad de Pedagogía, Universidad Panamericana, México, 2002, p. 255-271. 
